Symmetry-adapted molecular-field theory for thermotropic biaxial liquid crystals and its expansion at low temperature.

S.S. Turzi and T.J. Sluckin.

SIAM J. Applied Math. 73, 1139-1163 (2013)

(http://dx.doi.org/10.1137/120897237).

\title{
SYMMETRY ADAPTED MOLECULAR-FIELD THEORY FOR THERMOTROPIC BIAXIAL NEMATIC LIQUID CRYSTALS AND ITS EXPANSION AT LOW TEMPERATURE
}

\author{
S.S. TURZI* AND T.J. SLUCKIN ${ }^{\dagger}$
}

\begin{abstract}
We have analyzed some properties of mean field theories of thermotropic biaxial liquid crystals with $\left(D_{2 h}\right)$ symmetry. The study consists of two parts. In the first part we re-examine the standard theory due to Virga and coworkers. We introduce a convenient symmetry-adapted parameterization which allows the phase diagram to be displayed in an explicitly symmetry-preserving triangle. In the second part we use the Laplace approximation to examine the low temperature properties of the liquid crystal order parameters. Two of these (conventionally, $D, P$ ) are identically zero at zero temperature in a biaxially ordered phase, non-zero at low temperatures in the liquid crystalline phases, but are again zero at higher temperatures in the isotropic phase. We use a low temperature expansion to derive an expression for the dominant terms in the free energy. This functional is minimized to obtain the low temperature properties of the order parameters $D, P$.
\end{abstract}

Key words. liquid crystals, phase transitions, invariance and symmetry properties

AMS subject classifications. 76A15, 82D30, 82B26, 58J70

DOI.

1. Introduction. Daniel Vorländer [59] was the first to realize that that the presence of thermotropic nematic phase is strongly associated with a rod-like shape in the component molecules. The success of the Grandjean-Maier-Saupe molecular field picture $[30,48]$ confirmed the basic idea. In its most naive form, this theory supposes that a uniaxial liquid crystal phase is the consequence of cylindrically symmetric interactions between strictly uniaxial rod-like molecules. However, the molecules in chemists' test tubes stubbornly refuse to conform to theoretical idealizations. In fact mesogenic molecules tend to be, at the very least biaxial, with the two minor axes distinguished on a molecular length scale.

The presumed intimate relationship between the point symmetries of the molecules, on the one hand, and of the low temperature phases, on the other, gave rise to theoretical speculation about the possible existence of purely orientational biaxial liquid crystalline phases. In 1970 Freiser [26, 27] constructed a molecular field theory for a fluid of biaxial liquid crystal molecules, generalizing the Maier-Saupe theory [48]. He predicted that the usual first order isotropic - (uniaxial) nematic $\left(I-N_{u}\right)$ phase transition would be followed at lower temperatures by a second order uniaxial-biaxial nematic $\left(N_{u}-N_{B}\right)$ phase transition. In 1973 Alben [3] constructed a Landau theory which addressed some of the issues raised by Freiser. He predicted the existence of a point, later known as a Landau point, at which the isotropic-nematic phase transition became continuous, and at which the uniaxial-biaxial and isotropic-uniaxial nematic transitions collide. The Landau point, at which the biaxial nematic phase undergoes a second order transition to the isotropic phase, has been the focus of much subsequent study, and will be one of the subjects addressed in this paper.

An explicit example of biaxial molecular fluid is a fluid of hard biaxial particles with $\left(D_{2 h}\right)$ symmetry. In 1974 Straley [57] constructed a molecular field theory for this

\footnotetext{
*Università degli Studi eCampus, Via Isimbardi 10, 22060 Novedrate (CO), Italy (stefano.turzi@polimi.it).

${ }^{\dagger}$ Mathematics, Faculty of Social and Human Sciences, University of Southampton, Southampton, SO17 1BJ, UK.
} 
system, using an excluded volume parameterization. The predicted phase sequence agreed with that of Freiser and Alben. Straley realized that the statistical mechanics required not merely "uniaxial" and "biaxial" scalar order parameters, but also others which took account of the orientational ordering of the so-called "minor" molecular axes. The minimum set introduced by Straley (in his language $S, T, U, V$, and for which in this paper we use $S, P, D, C$ [22]) has come to be regarded as conventional, albeit that authors have not uniformly adopted the same normalization [54].

Yu and Saupe [60] observed a lyotropic biaxial phase in 1980, but the thermotropic biaxial phase has been much more elusive. In the latter case, despite intense study, and a number of positive reports [47, 2], many current observations remain controversial $[40,41]$. However, theoretical and simulation studies of the statistical mechanics of the biaxial phases are of some intrinsic interest, and also to clarify conditions required for fruitful experimental observations.

A key theme running through the theoretical literature has been the idea that as the degree of biaxiality increases, the isotropic-uniaxial nematic phase transition becomes more continuous. The conventional wisdom was as follows. First, the order parameter jump (howsoever defined) becomes less, the temperature gap $T_{N I}-T^{*}$ between the phase transition temperature $T_{N I}$ and a point of incipient thermodynamic divergence $T^{*}$ decreases, and the order parameter correlation length $\xi_{c}$ at $T_{N I}$ increases. At the same time there is a low temperature biaxial nematic phase with a continuous uniaxial-biaxial phase transition. The uniaxial-biaxial $\left(N_{U}-N_{B}\right)$ phase transition temperature $T_{U B}<T_{N I}$ increases as the degree of biaxiality is increased. This situation continues until a critical degree of biaxiality is reached, at which point $T_{U B}=T_{N I}$, and the (now direct isotropic-biaxial) phase transition becomes continuous $[3,13,10,51]$. This is the Landau point.

But beyond this degree of biaxiality, the original phase progression is restored. Some have identified this exchange as going from a rod-like to a disc-like molecular shape. Although we do not take this point of view, there is certainly an exchange of principal molecular orientations. As the degree of "biaxiality" (as originally conceived) is further increased, now the nematic-isotropic transition becomes more first order, and the uniaxial-biaxial transition decreases in temperature. Eventually a situation is reached at which the molecules are again uniaxial, but now the molecular axis of symmetry has changed. The biaxial phase disappears, and the uniaxial phase follows the predictions of the old theories.

However, although this picture appeared theoretically robust, there were some apparently discordant experimental observations in lyotropic systems $[53,60,4]$. These experiments, albeit in colloidal systems with some extra physics, suggested the possibility of direct first order transitions between isotropic and biaxial phases (rather than merely a Landau point), and of first order $N_{U}-N_{B}$ transitions. Thus although the result, due to Sonnet et al. $[56,12]$, that molecular field theory permits first order $I-N_{B}$ and $N_{U}-N_{B}$ transitions over a range of biaxiality parameters seemed to go against the canonical picture, it did resolve an experimental paradox.

This paper presents some theoretical results within the molecular field picture of nematic liquid crystals. In $\S 2$, we examine some aspects of the model developed in Refs. $[56,12]$. The traditional molecular field picture used the so-called geometric mean approximation for the effective intermolecular potential [46, 13, 39, 43]. This approximation, which reduces the number of molecular biaxiality parameters from two to one, is also implicit in much other work as well [3, 57]. The approximation is analogous to the well-known Lorentz-Berthelot mixing rule relating strength of the 
heteroatomic Van der Waals interaction to the strengths of the homoatomic interaction [49]. At first sight it seems unlikely that relaxing it will change qualitative features of the predicted phase diagram.

However, Sonnet et al. [56] developed a mean field picture in which the geometric mean approximation is drastically broken, albeit within a molecular picture that some might regard as remaining uniaxial. This model sustains a low temperature phase which can be identified with a biaxial phase. Moreover, although it offends naive ideas of what is meant by biaxiality, it has the distinct computational advantage of admitting a single scalar uniaxial order parameter and a single scalar biaxial order parameter. This model predicted, for suitable parameters, both first order $I-N_{B}$ and $N_{U}-N_{B}$ transitions. Furthermore, Sonnet et al. asserted this phase diagram to be universal, in that the key features of the phase diagram would be retained if some of their more drastic approximations were relaxed.

The more general study by Bisi et al. [12] more or less ratified this expectation. Now four rather than two order parameters were required for a full description, and the biaxiality is described by two independent parameters. The full phase diagram now could be represented in what these authors call "the essential triangle". The essentiality lies in a somewhat complicated set of transformations associated with exchange of molecular axes, causing physical information to be repeated if the whole biaxiality plane is considered. The purpose of our $\S 2$ is to use a picture based on traditional representations of phase diagrams of ternary mixtures, to visualize this information in what believe is a clearer manner.

This visualization is mainly concerned with the topological (global) properties of a phase diagram. But for both practical and theoretical reasons it is also important to be able to study the local properties of a phase diagram. To this end the Landau expansion of the free energy, in terms of low powers of the order parameters, is often a privileged route due to its simplicity. However, in its standard form, this procedure does not provide an accurate method with which to study the highly ordered phases. Specifically, the details of the dependence of the order parameters on temperature far from the onset of nematic order are not accessible from a Landau expansion.

In Sec. 3 we develop a method to construct an approximation of the free energy in the low temperature régime. This method goes beyond the usual Landau picture, but nevertheless maintains the key idea of expansion in terms of order parameters. The procedure is simple enough to be able to perform a semi-analytical analysis. However, although it is only approximate, it nevertheless yields accurate results just where the phases are most highly ordered. In particular, we can explore the low temperature properties of the order parameters as a function of the degree of biaxiality. Finally in $\S 4$ we draw some brief conclusions.

\section{Symmetry-preserving phase diagram.}

2.1. Order parameters and internal energy. We consider a fluid made from biaxial molecules with $\left(D_{2 h}\right)$ point group symmetry. Let $\left(\boldsymbol{m}_{1}, \boldsymbol{m}_{2}, \boldsymbol{m}_{3}\right)$ be unit vectors along the three principal molecular (symmetry) directions. These quantities represent what are usually known as the molecular axes. Define $\alpha, \beta$ and $\gamma$ to be the three Euler angles, describing the orientation of the molecular frame of reference with respect to the laboratory axes. There are only four independent scalar order parameters for a system composed of molecules possessing $\left(D_{2 h}\right)$ point group symmetry in a phase with the same symmetry (see e.g. [54]). These are conventionally denoted by $S, D$, 
$P$ and $C$ and are written in terms of the Euler angles as [54]:

$$
\begin{aligned}
S & =\frac{1}{2}\left\langle 3 \cos ^{2} \beta-1\right\rangle, \quad P=\frac{3}{2}\left\langle\cos 2 \gamma \sin ^{2} \beta\right\rangle \\
D & =\frac{3}{2}\left\langle\cos 2 \alpha \sin ^{2} \beta\right\rangle, \quad C=\frac{3}{2}\left\langle\left(1+\cos ^{2} \beta\right) \cos 2 \alpha \cos 2 \gamma-2 \cos \beta \sin 2 \alpha \sin 2 \gamma\right\rangle,
\end{aligned}
$$

where the angle brackets denote averaging over all possible orientations of the molecules.

In general, in the isotropic phase $(I)$, all order parameters vanish. In a phase with uniaxial order $\left(N_{U}\right), S$ and $D$ are non-zero, while $P=C=0$. However, in the more ordered biaxial phase $\left(N_{B}\right)$, all four order parameters are generally non-zero. We note that, in the limit of perfect ordering, we may suppose without loss of generality that the molecular and laboratory frames coincide. In this case, $\alpha, \beta, \gamma \rightarrow 0$, and it follows from the definitions in Eqs.(2.1-2.2) that $S \rightarrow 1, P \rightarrow 0, D \rightarrow 0$, and $C \rightarrow 3$.

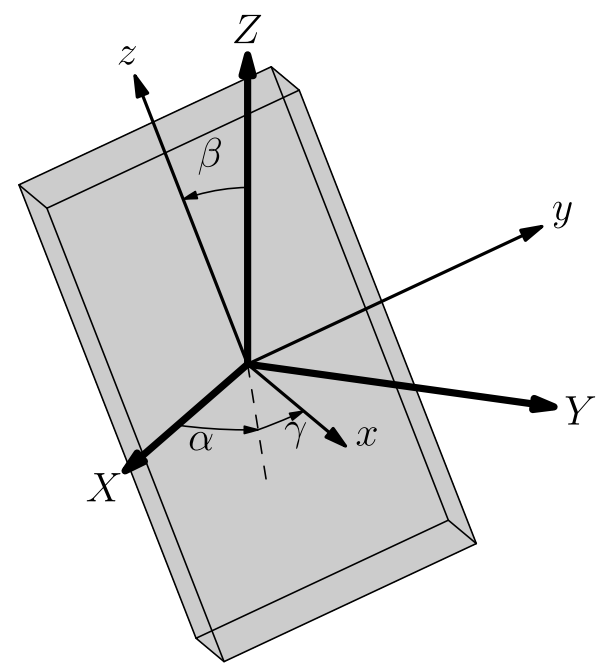

FIG. 2.1. Schematic description of a molecule possessing $\left(D_{2 h}\right)$ point group symmetry. The molecular and the laboratory frames of reference are identified by $(x, y, z)$ and $(X, Y, Z)$, respectively. The Euler angles $\alpha, \beta$, and $\gamma$ are also shown.

The order parameters $P$ and $D$ thus exhibit rather anomalous behavior. In the high temperature $I$ phase they vanish. In the $N_{U}$ phase, $D \neq 0$, and as temperature is further decreased into the $N_{B}$ phase, $P \neq 0$ also. But at sufficiently low temperatures, they vanish again, having gone through a maximum along the way. In theories of biaxial liquid crystals, $S$ and $C$ are often interpreted as principal order parameters, for increasing orientational order implies increasing values of these quantities. On the other hand, $D$ and $P$, which may not grow large, are regarded as subsidiary order parameters which are in some sense slaved by the principal order parameters, subject to the degree of molecular asymmetry. We shall return to this point in the next section.

The internal energy $U$ in the molecular-field theory is usually derived from a spherical tensor approach[46, 39, 42, 43]:

$$
U=-\frac{1}{2}\left[u_{200}\left(S^{2}+\frac{1}{3} P^{2}\right)+\sqrt{\frac{8}{3}} u_{220}\left(S D+\frac{1}{3} P C\right)+\frac{2}{3} u_{222}\left(D^{2}+\frac{1}{3} C^{2}\right)\right],
$$


where the $u_{2 m n}$ are related to coefficients in the spherical tensor expansion of an effective molecular interparticle potential. A full description of the relative molecular biaxiality requires two parameters, usually labeled $\lambda, \gamma$, where $\lambda=\frac{u_{222}}{u_{200}}$ and $\gamma=$ $\frac{u_{220}}{u_{200}}$. Eq.(2.3) corresponds, for example, to the molecular field approximation of the intermolecular potential proposed long ago by Straley [57]. The expansion of $U$ in Eq.(2.3) is the most general quadratic rotational invariant built from these order parameters [43].

The spherical tensor description is a powerful approach which, in particular, enables rotationally invariant and non-redundant expressions to be constructed without difficulty. On the other hand, a disadvantage of this approach is that the molecular $z$-axis has a privileged status from the beginning, as it is chosen as the long-axis of the molecules with preferred alignment to the laboratory $z$-axis. The privileged status assigned to one of the molecular axes and to the laboratory axis is necessary in order to perform practical calculations. But it introduces an artificial and arbitrary element which reflects on the subsequent developments of the theory and, clearly, has no physical significance.

A major theoretical goal is to study the phase behavior of the system with respect to variations of the relative molecular biaxiality. In general the direction of alignment generally depends (continuously) on the degree of molecular biaxiality. The physics of the system, however, must be independent of the labels assigned to the molecular and laboratory axis. Thus it is preferable not to privilege the status of one of the molecular or laboratory axes. Rather one should treat all the axes on an equal footing. In this case, the description of the system will be totally symmetric with respect to the relabeling of the axes.

As an example of difficulties that can emerge from this approach, let us consider the standard phase diagram of a biaxial nematic liquid crystal in the geometric mean approximation $[46,13,39,43]$. In this picture, molecular biaxiality is defined by the single parameter $\lambda$ in Eq.(2.3) above:

$$
\lambda=\frac{u_{222}}{u_{200}}=\left(\frac{u_{220}}{u_{200}}\right)^{2}=\gamma^{2} .
$$

The phase diagram resulting from this approximation, following the calculations of Boccara et al. [13] is plotted in Fig 2.2. The $N_{U}-N_{B}$ phase transition line exhibits a cusp at $\gamma=1 / \sqrt{6}$. For this value of $\gamma$ the aligning strength of the molecular $x$ and $z$ axes is equal, and the molecular biaxiality may be regarded as being maximal. The uniaxial nematic phase disappears, and there is now a direct $I-N_{B}$ phase transition. The phase diagram has some kind of mirror symmetry with respect to $\gamma_{c}=1 / \sqrt{6}$. The two sides of the phase diagram represent closely analogous physical situations. For each value of $\gamma_{1}<\gamma_{c}$, there is an associated value $\gamma_{2}>\gamma_{c}$ exhibiting equivalent physics, but with a rescaled fundamental energy parameter. Boccara et al. have asserted that $\gamma>\gamma_{c}$ corresponds to discs, whereas $\gamma<\gamma_{c}$ correspond to rods, and that this mapping indicates that the physics of rods and of discs is equivalent. Our picture is slightly different, but in any event, the relative molecular biaxiality $\gamma=0$ (and hence, by construction, uniaxial molecules) is formally and physically equivalent to $\gamma=\sqrt{3 / 2} \approx 1.2247$. In each case the theory describes a system of uniaxial $\left(D_{\infty h}\right)$ molecules. The only difference is that at $\gamma=\sqrt{3 / 2}$ the molecular $x$-axes align rather than the "natural" molecular $z$ - axes. The lack of linearity of the mapping over the range, and the fact that the value $\gamma=\sqrt{3 / 2}$ is not immediately obvious, suggests 


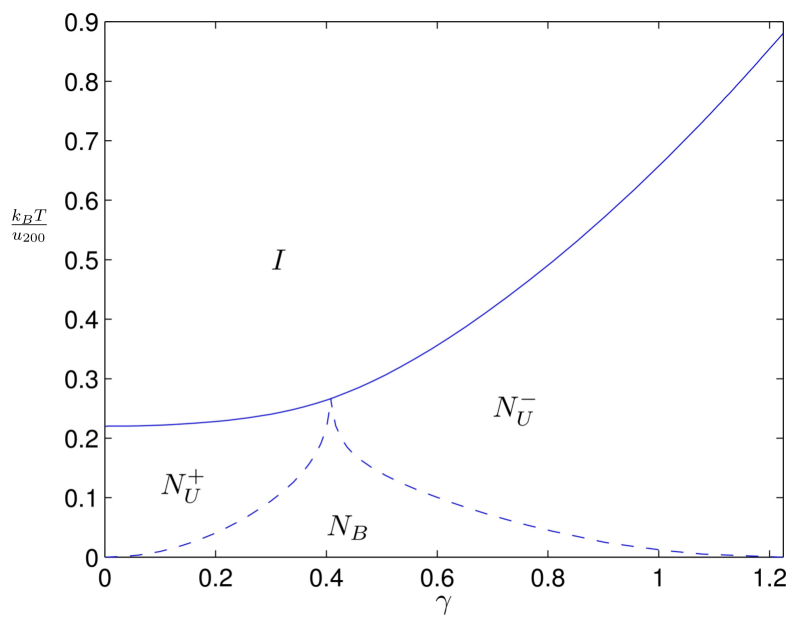

FIG. 2.2. Phase diagram after Boccara et al. [13]. Normalized temperature scale on y axis and biaxiality parameter $\gamma$ along $x$ axis. The two uniaxial phases $N_{U}^{+}$and $N_{U}^{-}$are associated with molecular alignment along molecular $z$ and $x$ axes respectively, and meet at a Landau point also coincident with a continuous transition from the biaxial nematic $N_{B}$ to the isotropic phase $I$. Note also the hint of some kind of symmetry between the regions with $\gamma$ less than and greater than $\frac{1}{\sqrt{6}}$.

that the parametrization of the molecular biaxiality, although useful for small degrees of biaxiality, is not optimal over the whole range.

There is an alternative, purely Cartesian, approach to the theory of biaxial nematic $\left(D_{2 h}\right)$ liquid crystals. This has been developed by Virga and colleagues $[56,12,20,21,11]$. These theories involve two ordering tensors $\boldsymbol{Q}$ and $\boldsymbol{B}$, defined as

$$
\boldsymbol{Q}=\left\langle\boldsymbol{m}_{3} \otimes \boldsymbol{m}_{3}-\frac{1}{3} \boldsymbol{I}\right\rangle, \quad \boldsymbol{B}=\left\langle\boldsymbol{m}_{1} \otimes \boldsymbol{m}_{1}-\boldsymbol{m}_{2} \otimes \boldsymbol{m}_{2}\right\rangle
$$

where, in the laboratory frame of reference

$\boldsymbol{Q}=\left(\begin{array}{ccc}-\frac{1}{3}(S-P) & 0 & 0 \\ 0 & -\frac{1}{3}(S+P) & 0 \\ 0 & 0 & \frac{2}{3} S\end{array}\right) ; \quad \boldsymbol{B}=\left(\begin{array}{ccc}-\frac{1}{3}(D-C) & 0 & 0 \\ 0 & -\frac{1}{3}(D+C) & 0 \\ 0 & 0 & \frac{2}{3} D\end{array}\right)$

and where $S, D, P, C$ are as defined in Eqs.(2.1,2.2) [54].

Their internal energy, as deduced from the mean-field potential, is

$$
\begin{aligned}
U & =-\frac{1}{2} \tilde{U}_{0}(\boldsymbol{Q} \cdot \boldsymbol{Q}+2 \Gamma \boldsymbol{Q} \cdot \boldsymbol{B}+\Lambda \boldsymbol{B} \cdot \boldsymbol{B}) \\
& =-\frac{\tilde{U}_{0}}{3}\left[\left(S^{2}+\frac{1}{3} P^{2}\right)+2 \Gamma\left(S D+\frac{1}{3} P C\right)+\Lambda\left(D^{2}+\frac{1}{3} C^{2}\right)\right],
\end{aligned}
$$

where $\Gamma$ and $\Lambda$ are the two measures of molecular biaxiality.

In the uniaxial liquid crystal, only the tensor $\boldsymbol{Q}$ contributes to the energy. The tensor $\boldsymbol{B}$ is associated with the difference in alignment properties between the molecular $x$ and $y$ axes, and in a uniaxial phase would be expected to be zero. As expected, the internal energy given in Eq.(2.7) turns out to be equivalent to that given in Eq.(2.3). We note here that although the theory has been expressed in apparently invariant 
tensor form, in practice, the privileged status of the molecular $z$ axis is nevertheless preserved.

We now develop a theory that avoids assigning a favored status to any of the molecular axes. The resulting formulae will retain some symmetry lost by privileging the molecular $z$ axis. We first note that a distinct ordering tensor is associated with each principal molecular axis $[61,58]$. This leads to the the definition of the three symmetric traceless tensors

$$
\boldsymbol{Q}_{1}=\left\langle\boldsymbol{m}_{1} \otimes \boldsymbol{m}_{1}-\frac{1}{3} \boldsymbol{I}\right\rangle, \quad \boldsymbol{Q}_{2}=\left\langle\boldsymbol{m}_{2} \otimes \boldsymbol{m}_{2}-\frac{1}{3} \boldsymbol{I}\right\rangle, \quad \boldsymbol{Q}_{3}=\left\langle\boldsymbol{m}_{3} \otimes \boldsymbol{m}_{3}-\frac{1}{3} \boldsymbol{I}\right\rangle .
$$

The three tensors $\boldsymbol{Q}_{i}$ each describe the orientation of one molecular axis with respect to the laboratory axis, replacing the two tensors $\boldsymbol{Q}, \boldsymbol{B}$. We note that, by construction, the three $\boldsymbol{Q}_{i}$ are not independent but must satisfy $\boldsymbol{Q}_{1}+\boldsymbol{Q}_{2}+\boldsymbol{Q}_{3}=0$. The replacement of the two independent tensors $\boldsymbol{Q}, \boldsymbol{B}$ by the three dependent tensors $\boldsymbol{Q}_{i}$ has the disadvantage that the dependence must be borne in mind, but the advantage that no molecular axis has a special status.

In a phase with $D_{2 h}$ symmetry, the eigenframes of the three tensors $\boldsymbol{Q}_{i}$ will be mutually diagonalizable. The laboratory axes are then chosen to be coincident with this common eigenframe in order to minimize the number of independent scalar order parameters. The internal energy can now be rewritten in terms of the standard quartet of order parameters as follows:

$$
\begin{aligned}
U & =-\frac{1}{2} U_{0}\left(\lambda_{1} \boldsymbol{Q}_{1} \cdot \boldsymbol{Q}_{1}+\lambda_{2} \boldsymbol{Q}_{2} \cdot \boldsymbol{Q}_{2}+\lambda_{3} \boldsymbol{Q}_{3} \cdot \boldsymbol{Q}_{3}\right) \\
& =-\frac{U_{0}}{3}\left[\left(\frac{\lambda_{1}+\lambda_{2}}{4}+\lambda_{3}\right)\left(S^{2}+\frac{1}{3} P^{2}\right)+\frac{1}{2}\left(\lambda_{2}-\lambda_{1}\right)\left(S D+\frac{1}{3} P C\right)\right. \\
& \left.+\frac{1}{4}\left(\lambda_{1}+\lambda_{2}\right)\left(D^{2}+\frac{1}{3} C^{2}\right)\right] .
\end{aligned}
$$

In this parameterization, the overall strength of the interaction is parameterized by $U_{0}$. To determine the topology of the phase diagram, only the relative strength of the different orientational tensor interactions is important, and the constraint $\lambda_{1}+$ $\lambda_{2}+\lambda_{3}=1$ can be assumed without loss of generality.

From Eqs.(2.3) and (2.9), explicit expressions for the transformations for the molecular biaxiality parameters can be written as:

$$
\left\{\begin{array} { l } 
{ u _ { 2 0 0 } = \frac { U _ { 0 } } { 6 } ( \lambda _ { 1 } + \lambda _ { 2 } + 4 \lambda _ { 3 } ) } \\
{ u _ { 2 2 0 } = \frac { U _ { 0 } } { 2 \sqrt { 6 } } ( \lambda _ { 2 } - \lambda _ { 1 } ) } \\
{ u _ { 2 2 2 } = \frac { U _ { 0 } } { 4 } ( \lambda _ { 1 } + \lambda _ { 2 } ) }
\end{array} \Leftrightarrow \left\{\begin{array}{l}
\lambda_{1}=\left(2 u_{222}-\sqrt{6} u_{220}\right) / U_{0} \\
\lambda_{2}=\left(2 u_{222}+\sqrt{6} u_{220}\right) / U_{0} \\
\lambda_{3}=\left(3 u_{200}-2 u_{222}\right) / 2 U_{0},
\end{array}\right.\right.
$$

while a comparison between Eqs. (2.7) and (2.9) yields:

$$
\left\{\begin{array} { l } 
{ \tilde { U } _ { 0 } = \frac { U _ { 0 } } { 4 } ( \lambda _ { 1 } + \lambda _ { 2 } + 4 \lambda _ { 3 } ) } \\
{ \Gamma = \frac { \lambda _ { 2 } - \lambda _ { 1 } } { \lambda _ { 1 } + \lambda _ { 2 } + 4 \lambda _ { 3 } } } \\
{ \Lambda = \frac { \lambda _ { 1 } + \lambda _ { 2 } } { \lambda _ { 1 } + \lambda _ { 2 } + 4 \lambda _ { 3 } } }
\end{array} \Leftrightarrow \left\{\begin{array}{l}
\lambda_{1}=2 \tilde{U}_{0}(\Lambda-\Gamma) / U_{0} \\
\lambda_{2}=2 \tilde{U}_{0}(\Lambda+\Gamma) / U_{0} \\
\lambda_{3}=\tilde{U}_{0}(1-\Lambda) / U_{0}
\end{array} .\right.\right.
$$

By imposing the constraint $\lambda_{1}+\lambda_{2}+\lambda_{3}=1$ we also derive the identity: $U_{0}=$ $\tilde{U}_{0}(1+3 \Lambda)$. 


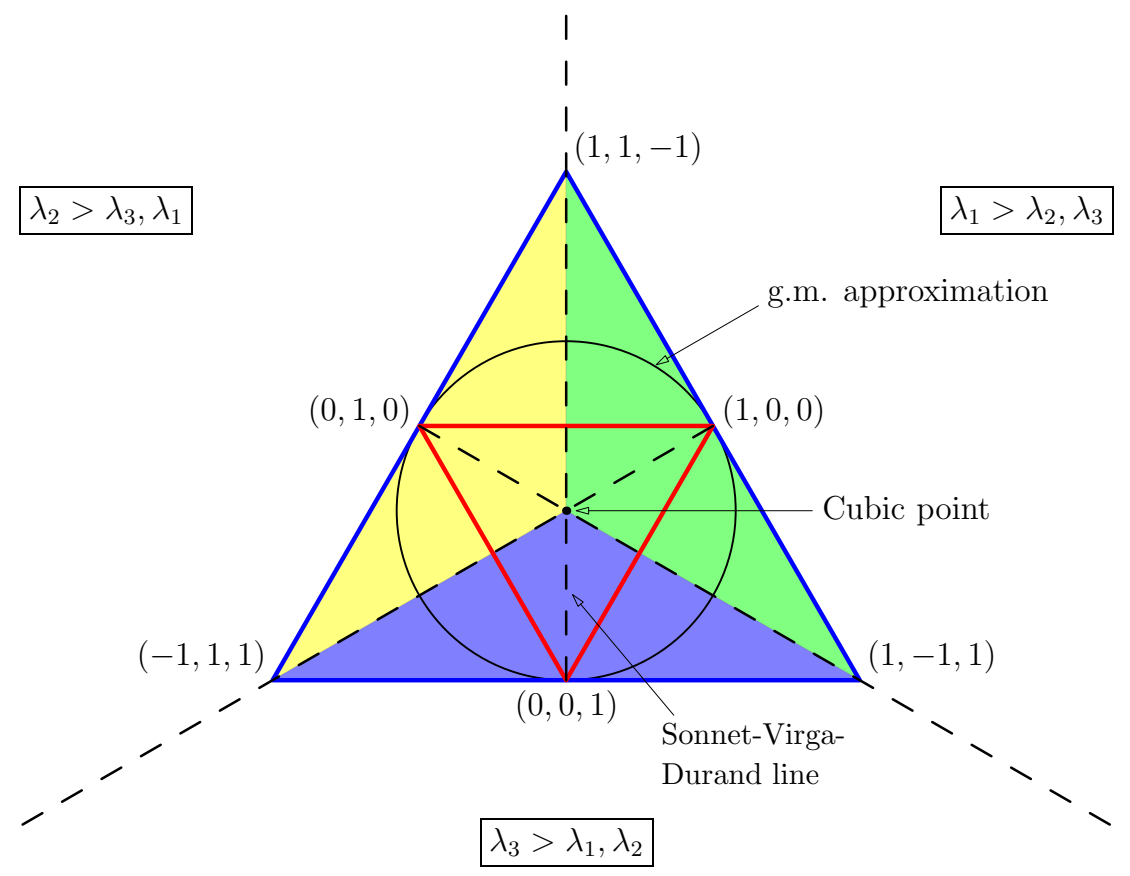

FIG. 2.3. Ternary diagram of the ground states. The center of the diagram is defined by the cubic point whose coordinates are $\left(\frac{1}{3}, \frac{1}{3}, \frac{1}{3}\right)$. The dashed lines separate the plane into three sectors in which the dominant attractive anisotropic interaction is, respectively, along the $z$ (blue), $y$ (yellow) and $x$ (green) molecular axis. These sectors meet at a common vertex known as the cubic point. The inner triangle (shown with red edges) encloses those points for which all the axes interact attractively. The external triangle (shown with blue edges) identifies the limit of stability of the biaxial phase. The geometric mean approximation, as given in Eq.(2.14), corresponds to a circle passing through the vertices of the red triangle, with center at the cubic point.

2.2. Ground state ternary diagram. The principal advantage of the $\left(\lambda_{1}\right.$, $\left.\lambda_{2}, \lambda_{3}\right)$ representation is that the degree of biaxiality is represented in terms of a barycentric coordinate system. One related context in chemical physics is that of complex fluids; concentrations in a ternary mixture are often expressed using this picture. Phase diagrams in ternary mixtures require a discussion of phases at particular relative concentrations $\left\{c_{i}\right\} ; c_{1}+c_{2}+c_{3}=1$. These concentrations can be represented as points in an equilateral triangle [55]. One picture of this triangle is to think of the relative concentrations as represented by points $\left(c_{1}, c_{2}, c_{3}\right)$ in ordinary three-dimensional Cartesian space but constrained to lie on the plane $c_{1}+c_{2}+c_{3}=1$.

In ternary mixtures, pure systems (e.g. $c_{1}=1, c_{2}=c_{3}=0$ ) are then represented by vertices of the equilateral triangle. Two-component submixtures map onto the edges of the triangle. The region of all-positive relative concentrations is then the equilateral triangle, with side $\sqrt{2}$ and height $\sqrt{3 / 2}$, and vertices at the points $(1,0,0)$, $(0,1,0)$ and $(0,0,1)$. As concentrations are never negative, points outside the concentration triangle are unphysical. The barycentric coordinate representation also crops up in a number of other mathematical contexts [16].

There is an immediate analogy to degrees of biaxiality, with the relative orientational alignment strengths $\lambda_{i}$ playing the role of the concentrations $c_{i}$. We shall refer to the set of points in the corresponding ternary diagram as the biaxiality parameter plane, which is shown in Fig.2.3. Uniaxial systems correspond to $\lambda_{i}=1$, for $i=1,2,3$, 
depending on whether the $x, y$ or $z$ molecular axes align. Two-component systems correspond to nematic systems in which two molecular axes tend to align, but the third is inert. The central point inside the biaxiality parameter plane, with coordinates $\left(\frac{1}{3}, \frac{1}{3}, \frac{1}{3}\right)$ we shall label as the cubic point. In this case the alignment properties of each molecular axis are the same, and the system will be expected to possess $O_{h}$ symmetry. However, by contrast with the ternary mixture, points outside the triangle do now map onto physical systems. If one, or even two, of the $\lambda_{i}$ are negative, this indicates that some molecular axes favor a mutual antiparallel alignment. However, in this picture the physical case in which all axes carry an antiparallel tendency requires a new diagram, because the constraint that $\sum \lambda_{i}=1$ is necessarily violated.

The phase diagram as a function of the biaxiality is found by studying the minima of the internal energy (2.9) as a function of the changing biaxiality parameters $\lambda_{1}, \lambda_{2}$ and $\lambda_{3}$. As described in detail elsewhere [43, 18, 14], each physical significant phase can be described by thirty-six equivalent choices of the order parameters $S, P$, $D$ and $C$. In a system with $D_{2 h}$ symmetry, this multiplicity of the order parameters is due to the physical irrelevance of the specific labels associated with the molecular and laboratory axes. In the same way, there are also six possible values of the set $\left(\lambda_{1}, \lambda_{2}, \lambda_{3}\right)$, related by a different choice of the axis labels, where the physical behavior of the liquid crystals is equivalent. These discrete symmetries correspond to triangular symmetries of the phase diagram in the biaxiality parameter plane.

We now discuss Fig.2.3 in more detail. For practical purposes it is also helpful to introduce an orthogonal frame of reference adapted to this plane, $\left(\gamma_{1}, \gamma_{2}, \gamma_{3}\right)$, with origin in the cubic point $\left(\lambda_{1}, \lambda_{2}, \lambda_{3}\right)=(1 / 3,1 / 3,1 / 3)$. Explicitly, the following change of variables

$$
\left\{\begin{array} { l } 
{ \gamma _ { 1 } = \frac { 1 } { \sqrt { 2 } } ( \lambda _ { 1 } - \lambda _ { 2 } ) } \\
{ \gamma _ { 2 } = \frac { 1 } { \sqrt { 6 } } ( \lambda _ { 1 } + \lambda _ { 2 } - 2 \lambda _ { 3 } ) } \\
{ \gamma _ { 3 } = \frac { 1 } { \sqrt { 3 } } ( \lambda _ { 1 } + \lambda _ { 2 } + \lambda _ { 3 } - 1 ) }
\end{array} \Leftrightarrow \left\{\begin{array}{l}
\lambda_{1}=\frac{1}{3}+\frac{\gamma_{1}}{\sqrt{2}}+\frac{\gamma_{2}}{\sqrt{6}}+\frac{\gamma_{3}}{\sqrt{3}} \\
\lambda_{2}=\frac{1}{3}-\frac{\gamma_{1}}{\sqrt{2}}+\frac{\gamma_{2}}{\sqrt{6}}+\frac{\gamma_{3}}{\sqrt{3}} \\
\lambda_{3}=\frac{1}{3}-\sqrt{\frac{2}{3}} \gamma_{2}+\frac{\gamma_{3}}{\sqrt{3}}
\end{array}\right.\right.
$$

takes the plane $\lambda_{1}+\lambda_{2}+\lambda_{3}=1$ into the coordinate plane $\gamma_{3}=0$. As can be easily checked, the transformation (2.12) is simply the composition of a translation and a rotation. As such, it preserves lengths and angles. The coordinates $\gamma_{1}$ and $\gamma_{2}$ play the role of the Cartesian coordinates $x$ and $y$ in the biaxiality parameter plane, with origin in the cubic point.

To obtain a complete phase diagram, a temperature axis must be introduced, orthogonal to the biaxiality parameter plane and therefore orthogonal to the picture in Fig.2.3. Each point in the figure can be interpreted as the projection of the phase diagram along the temperature axis. As a two-dimensional projection of a threedimensional phase diagram, the information on the actual transition temperatures is lost. It is possible nevertheless to represent the phase sequences.

However, we postpone the discussion of the presentation of the phase sequences and first concentrate on the description of the ground states of the system, i.e., the minima of the internal energy (2.9) as the biaxial parameters are varied. These fundamental states can be interpreted as the preferred phases when the entropy contribution to the free energy becomes negligible. In limiting the scope of the description, at least initially, we can easily identify the regions of the biaxial parameters plane with equivalent physical significance. By construction, the fact that our parametrization does 
not favor any axis, and thus respects the triangular symmetry of the system, plays a key role in the analysis.

The ground states depend only on the relative values of the $\left\{\lambda_{i}\right\}$. The plane can thus be divided into three separate but equivalent regions using the criteria that $\lambda_{1}>\lambda_{2}, \lambda_{3} ; \lambda_{2}>\lambda_{1}, \lambda_{3}$ or $\lambda_{3}>\lambda_{1}, \lambda_{2}$. In the specific case when $\lambda_{3}>\lambda_{1}, \lambda_{2}$, for example, the $z$-molecular axes will align most strongly, and tend to align first as the temperature is decreased.

The inner triangle (shown with red edges in Fig.2.3) is defined by the convex hull of the points $(1,0,0),(0,1,0)$ and $(0,0,1)$. Its sides are obtained by imposing $\lambda_{1}=0, \lambda_{2}=0$ and $\lambda_{3}=0$. Points inside this triangle are defined by positive values of the parameters $\lambda_{i}$ in the biaxiality parameter plane in Eq.(2.9). Physically, the anisotropic interactions between the molecule axes are all attractive. Thus, the ground state is here strongly biaxial, as all the molecular axes tend to align with each other, and by convention also with the laboratory axes.

We contrast the inner triangle with the outer triangle (shown with blue edges in Fig. 2.3), defined as the convex hull of the points $(1,1,-1),(1,-1,1)$ and $(-1,1,1)$. The sides of this triangle are determined by the equations $\lambda_{1}=1, \lambda_{2}=1$ and $\lambda_{3}=1$. The region between these two triangles contains points in the biaxiality parameter plane where the anisotropic interactions between the molecule axes are partly repulsive. Physically, this means that one of the $\lambda_{i}$ is negative, but the other two remain positive. However, as long as this repulsive interaction is weak, the ground state is still biaxial, although in some sense not "strongly biaxial". Now the transition to a biaxial nematic phase is expected to appear at a lower temperature. We expect the whole blue triangle to define the region of biaxial ground states, as we now show.

Without loss of generality, we can specifically consider an attractive interaction between the $z$-molecular axes. In this case the internal energy (2.9) attains its minimum when the molecular long-axes are aligned, and we can consider this to be oriented along the $z$-laboratory axis.

In a completely ordered phase, by definition (see Eqs. $(2.1,2.2)$ ), the secondary order parameters, $P$ and $D$, must vanish. The magnitude of the internal energy in a biaxial phase, as given in Eq.(2.9), can now be simplified, yielding:

$$
U_{b} \approx-\frac{U_{0}}{3}\left(\frac{1+3 \lambda_{3}}{4} S^{2}+\frac{1}{12}\left(\lambda_{1}+\lambda_{2}\right) C^{2}\right) .
$$

To determine whether a biaxial nematic state is favored we compare the absolute minimum of the internal energy in a biaxial nematic phase with that of a purely uniaxial nematic phase. When $\lambda_{3} \geq 1$ the sum $\lambda_{1}+\lambda_{2}$ must be lower or equal to zero. In this case, a minimization of $U_{b}$ in (2.13) when $\lambda_{3} \geq 1$ necessarily yields $C=0$. Thus a uniaxial internal energy has a lower energy, and thus the biaxial ground state is unstable.

The explicit triangular symmetry of the internal energy with respect to the relabeling of the axes then allows us to prove that the same conclusion holds also when we consider alignment with respect to the $x$ or $y$ axes. At first sight it would appear that the theory is predicting a uniaxial ground state. This is anomalous in that the uniaxial ground state for this system retains non-zero entropy, and corresponds to an orientational glass phase. However, in a continuous system, the region of liquid stability is usually relatively restricted, and at low temperatures the liquid state is preempted by a crystal, the details of which require a more detailed study of the spatial dependence of the interparticle potential. 
On the other hand, the orientational aspects of liquid crystalline behavior are often studied using nearest neighbor lattice models. The classic such model only deals with uniaxial interactions and is due to Lebwohl and Lasher [38]. The molecular field theory of such a model coincides with the Maier-Saupe theory [48], while the critical properties are anomalous in the same manner as experimental liquid crystals [23]. In the biaxial context, such a lattice was introduced by Biscarini et al. [10].

If the mean field model is interpreted as representing the properties of such a model, then the loss of stability of the biaxial ground state occurs whenever one of the $\lambda_{i}$ is greater than one. In Fig.2.3 this corresponds to the region outside the blue triangle. We interpret this as a possible instability of the uniform biaxial state with respect to a different ground state. Here, depending on the nature of the lattice, we expect the ground state to be either glassy or non-uniform. If the lattice is frustrated, the ground state will be degenerate and hence glassy [32]. If it is not frustrated, on the other hand, a case which obtains when the lattice is, for example, cubic, the ground state can be ordered. In general we expect a nematic analogue of an antiferromagnetic phase, characterized by nematic ordering along the principal axis, and anti-nematic ordering in sublattices along the other molecular axes. These systems have been studied by De Matteis and Romano [19]. We label this state as an antibiaxial phase.

Close to the edges of the triangle there is a competition between uniaxiality and biaxiality. The edges form multiphase lines, along which the ground state is extremely degenerate. We note that, as can be checked directly using the transformations (2.11), the blue triangle corresponds exactly to the region of stability labeled as the "essential triangle" in Ref.[56, 20, 12]. This region is defined by $\Lambda \geq 0 ; \Lambda-2|\Gamma|+1 \geq 0$. It is shown in Ref. [56, 20,18], with greater rigor than observed here, that only within this region, the state of complete alignment of two molecules is a non-degenerate minimum for the given mutual orientational interaction energy.

Usually it is complicated to draw three dimensional phase diagrams. In the literature of biaxial liquid crystals there are some traditional approximations that reduce the number of independent molecular biaxial parameters from two to one, so that the phase diagram can be represented on a two-dimensional plot. The first standard approximation is the geometric mean approximation discussed above. It is so-called because in the spherical tensor approach the relative molecular biaxiality coefficient $u_{220}$ is expressed as $u_{220}=\sqrt{u_{200} u_{222}}$. It has been noted this expression is consistent with the molecular interactions originating from London dispersion forces $[26,27,31,46,13]$. In Refs. [56, 21, 12], this approximation is called the "dispersion parabola", given by the equation $\Lambda=\Gamma^{2}$.

Substituting $u_{220}=\sqrt{u_{200} u_{222}}$, into Eqs.(2.10) yields the following relation in terms of $\lambda_{1}, \lambda_{2}, \lambda_{3}$ :

$$
\frac{1}{\lambda_{1}}+\frac{1}{\lambda_{2}}+\frac{1}{\lambda_{3}}=0
$$

A little algebra and the use of the adapted Cartesian coordinates $\left(\gamma_{1}, \gamma_{2}\right)$, as introduced in Eq.(2.12), transforms Eq.(2.14) into

$$
\gamma_{1}^{2}+\gamma_{2}^{2}=\frac{2}{3}
$$

Thus, in the biaxiality parameter plane, the geometric mean approximation corresponds to a circle, centered at the cubic point, and with radius $\sqrt{\frac{2}{3}}$. This geometric mean circle passes through the vertices of the red triangle and is shown in Fig.2.3. 
An alternative standard reduction of the number of molecular biaxiality coefficients is obtained by imposing $u_{220}=0$ (or $\Gamma=0$ ), which we refer to as the Sonnet-Virga-Durand approximation [56, 21]. As shown in $[21,12]$, this reduces the complexity of the theory by retaining just the two dominant order parameters, $S$ and $C$. An extension of this approximation for biaxial nematics with $C_{2 h}$ point group symmetry is considered in [44]. In the biaxiality parameter plane, the curve $u_{220}=0$ corresponds to the straight line defined by $\lambda_{1}=\lambda_{2}$. As is evident by the triangular symmetry, analogous approximations are obtained by setting $\lambda_{1}=\lambda_{3}$ or $\lambda_{2}=\lambda_{3}$. These approximations are shown as dashed lines in Fig.2.3.

It is now clear from Fig.2.3 that a single portion of the biaxiality parameter plane suffices to describe all physically relevant situations. For instance, the whole plane is a replica of the lower right triangular region with the vertex in the cubic point and delimited by the dashed lines identified by $\lambda_{1}=\lambda_{2}$ and $\lambda_{1}=\lambda_{3}$. Other portions of the biaxiality parameter plane are isomorphically and isometrically mapped into this fundamental region by exploiting the triangular and mirror symmetry of Fig.2.3.

The fact that a calculation of the full phase diagram requires only a partial covering of the biaxial parameter space is not new. In fact, this fundamental region is equivalent to the essential triangle introduced by Bisi et al. in Ref.[12]. However, the new feature is that in our case this result is an obvious consequence of the symmetry of the system, while in Ref.[12] the equivalent regions are greatly deformed and therefore their equivalence is less transparent and less suited to a practical exploitation of the intrinsic symmetries of the system.

2.3. Phase sequences. Fig. 2.3 provides us with a great deal of information in a compact and intuitive manner. However, it mainly describes the ground state of the liquid crystal, namely the phase of the highest order where the internal energy attains its global minimum. A more comprehensive picture is obtained when each point of Fig.2.3 is associated to a phase sequence, rather than a unique high order phase.

For instance, whilst the isotropic to uniaxial $\left(I-N_{U}\right)$ transition is always first order, it known that the uniaxial to biaxial $\left(N_{U}-N_{B}\right)$ transition can be either first or second order $[56,21,20]$. Thus, the $N_{U}-N_{B}$ transition line in the phase diagram may bear a tricritical point. Correspondingly, a tricritical line can be drawn in the biaxiality parameter plane, which divides it into two regions, according to whether the secondary uniaxial to biaxial transition is first or second order. Moreover, a triple line can be drawn, corresponding to the points where the isotropic, uniaxial, and biaxial phases are in equilibrium. More precisely, we can distinguish the region where there is a direct transition from the isotropic phase, $I$, to the biaxial phase, $N_{B}$, from that where there is an intermediate uniaxial nematic phase, $N_{U}$, by assigning different colors. The results of Refs. [56, 21, 20] show that in the Sonnet-Virga-Durand approximation, the transition from $N_{U}$ to $N_{B}$ phase can be either first or second order, thus evidencing the existence of a tricritical point. A second tricritical point is found at a higher temperature and a higher relative molecular biaxiality, along the line of direct transition from the isotropic to the $N_{B}$ phase. Furthermore, the lines of the first order transition $I-N_{U}$ and $N_{U}-N_{B}$ meet at a triple point. Thus, by symmetry in our ternary phase diagram we expect to be able to represent six tricritical points and three triple points along the dashed lines. An elementary conjecture is that these special points along the dashed lines are connected by a line of triple points and a line of tricritical points.

Using Eqs.(2.11), it is possible to rephrase the whole "universal mean-field phase diagram" and all the results reported in Refs.[56, 21, 20, 12, 11] in terms of $\lambda_{1}, \lambda_{2}$ and 
$\lambda_{3}$. The resulting phase sequences can be re-expressed in our biaxiality parameter plane. The transposition of these results, in the region inside the geometric mean approximation circle, is drawn in Fig.2.4. We remark that the quantitative plot of the tricritical line and the triple lines shown in Fig.2.4 requires a bifurcation analysis of the order parameters obtained from the minimization of the molecular field free energy. The numerical data contained in Fig.2.4 are extracted from Ref.[12].

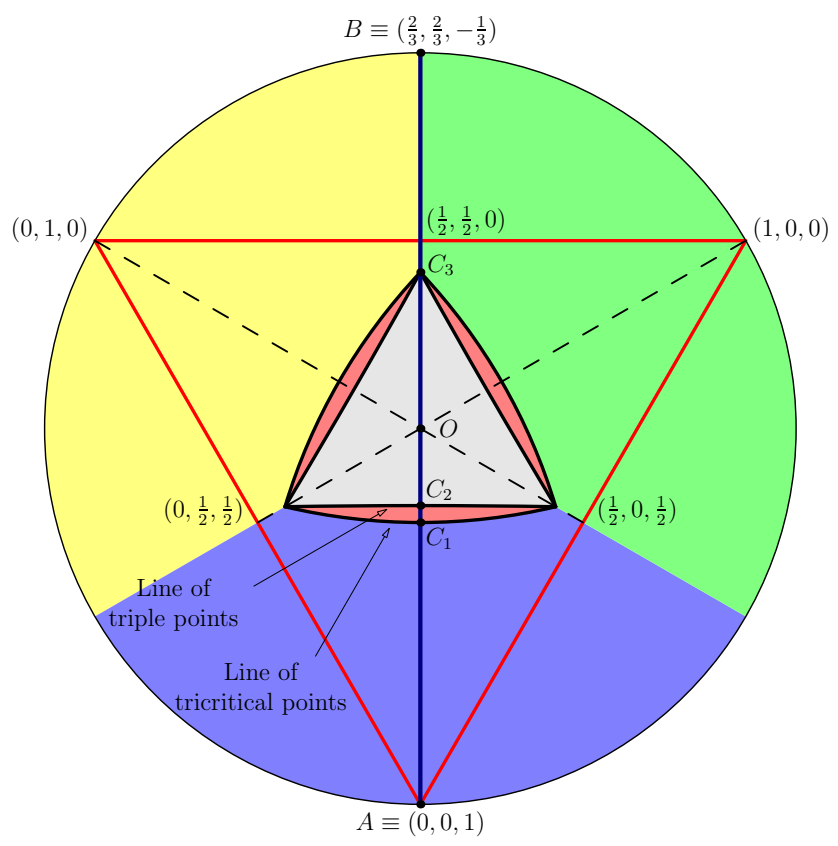

FIG. 2.4. Ternary diagram of the phase sequences, limited to the region of the biaxiality parameter plane enclosed by the geometric mean circle. The point $C_{1}$ represents a tricritical point, $C_{2}$ is a triple point and $C_{3}$ is a Landau critical end point. The different phase sequences are separated by a line of tricritical points and a line of triple points.

To improve the description of the regions with different phase sequences, we explore Fig.2.4. We start at the point $A \equiv(0,0,1)$, proceed along the SonnetVirga-Durand line $\lambda_{1}=\lambda_{2}$ (shown as a solid deep blue line), to reach the point $B \equiv\left(\frac{2}{3}, \frac{2}{3},-\frac{1}{3}\right)$. We remark that both $A$ and $B$ lie on the geometric mean circle. As already mentioned in Sec.2.2, the strongest anisotropic interaction in the blue, yellow and green sectors are, respectively, along the molecular $z, y$ and $x$ axes.

As already noticed, at $A$ the biaxial phase $N_{B}$ is unstable, and there is only a first order transition from the isotropic to the uniaxial nematic phase: $I-N_{U}$. In what follows, we adopt the convention of a single dash to denote a first order transition, while we reserve a double dash, $=$, for a second order transition. Along the segment $A C_{1}$, a biaxial nematic phase appears and the phase sequence is: $I-N_{U}=N_{B}$. The same phase sequence is expected within the blue sector, and by symmetry, also within the yellow and green sectors, although in these last two regions it will be the molecular $y$ or $x$-axes which align in the intermediate uniaxial phase. We emphasize the phase sequence $I-N_{U}=N_{B}$ is valid only in the inner part of the sectors and not along the edges between two sectors. We postpone the discussion of this interesting point when we study the phase behavior along the segment $C_{3} B$.

The point $C_{1}$ represents a tricritical point. Here the continuous transition $N_{U}=$ 
$N_{B}$ becomes first order. Therefore the phase sequence in the tiny red regions is $I-N_{U}-N_{B}$. As we move towards the cubic point, we hit the triple point $C_{2}$ where all the three phases, $I, N_{U}$ and $N_{B}$ coexist. As we move further the anisotropic interactions of the molecular axis become comparable and the molecules want to align along all the axes as soon as the temperature is low enough to abandon the isotropic phase. Therefore, in the grey region there is a direct first order transition to the $N_{B}$ phase: $I-N_{B}$. The point $C_{3}$ is where the line of triple points and the line of tricritical points meet. We call this a Landau critical end point.

At $C_{3}$ the transition to the $N_{B}$ phase is still direct from the isotropic phase, but becomes continuous, while at the same time the region over which the direct transition occurs shrinks to a single line. Furthermore, the same phase sequence, $I=N_{B}$, is also observed along the whole segment $C_{3} B$.

It must be noted, however, that along the segment $O B$ the interaction of the molecular $z$-axes is weakly attractive or weakly repulsive (depending on the sign of $\lambda_{3}$ ), whereas the interactions of the $x$ and $y$-axes are equal and strongly attractive. Therefore, the uniaxial phase is never favored since there is not a single preferred direction of alignment. By contrast, the biaxial phase is possible since the strong attractive interaction of the $x$ and $y$ axes forces also the $z$ axes to align. Slightly away from the segment $C_{3} B$, thus breaking the symmetry of the $x$ and $y$ axes, the $N_{U}$ phase appears, although the transition $I-N_{U}$ is expected to be very weak. This result is confirmed by the phase diagrams calculated along the geometric mean approximation $[57,13,43]$, where the transition $I-N_{U}$ becomes weaker and weaker as the point $B$ is approached.

As a final application, we are now in a position to show how the geometric mean phase diagram, plotted in Fig.2.2, is transformed when we employ our parametrization $\lambda_{1}, \lambda_{2}$ and $\lambda_{3}$. With reference to Fig.2.4, the geometric mean line corresponds, in our biaxiality parameter plane, to a circle centered at the cubic point, and with radius $\sqrt{\frac{2}{3}}$. In order to study the phase behavior along this line, we use the azimuthal coordinate $\vartheta$ which measures the counter-clockwise angle starting from the point $A \equiv(0,0,1)$. With the aid of Eqs.(2.10) and (2.12) it is possible to show that

$$
\begin{aligned}
\gamma & =\frac{u_{220}}{u_{200}}=\frac{1}{\sqrt{2}} \tan \frac{\vartheta}{2}, \\
\tau & =\frac{k_{B} T}{u_{200}}\left(\frac{1+\cos \vartheta}{3}\right) .
\end{aligned}
$$

The route between $(0,0,1)(\vartheta=0)$ and $(1,0,0)\left(\vartheta=\frac{2 \pi}{3}\right)$ corresponds to a progressive exchange of nematic interaction strength between $z$ and $x$ molecular axes, subject to the geometric mean approximation. This formulation now explicitly ensures that the transition temperatures are equal if $\lambda_{1}$ and $\lambda_{3}$ are exchanged. The route corresponds to the range $\left[0, \frac{2 \pi}{3}\right]$.

These transformations map the phase diagram of Boccara et al.[13] shown in Fig.2.2 into a different but physically equivalent form shown in Fig.2.5. The diagram is now explicitly symmetric around $\vartheta=\frac{\pi}{3}$, as one expects from the analytic formulation. In our picture $\vartheta=0$ i.e. $\lambda_{3}=1$, corresponds to nematic rods whose principal axis lies along the molecular $z$ axis, while $\vartheta=\frac{2 \pi}{3}$ i.e. $\lambda_{1}=1$ corresponds to nematic rods whose principal axis lies along the molecular $x$ axis. This contrasts with the point 


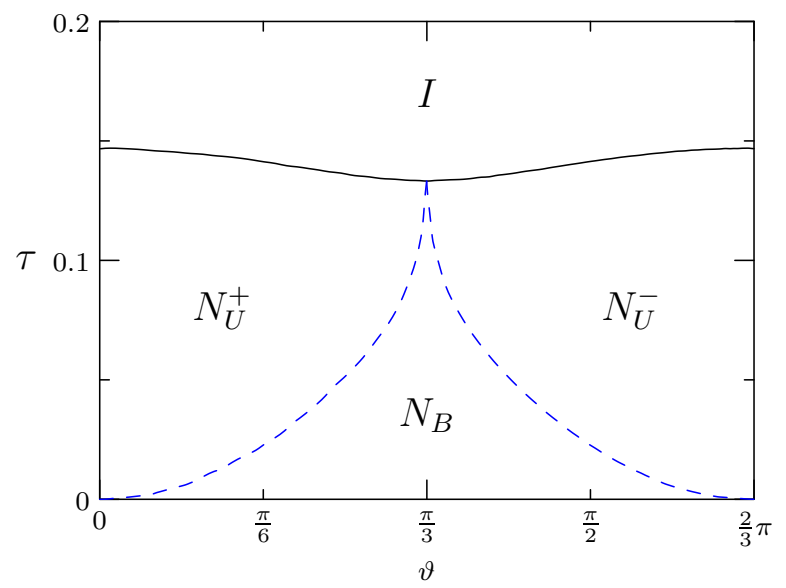

FIG. 2.5. Symmetric form of the geometric mean phase diagram, obtained using the biaxiality parameters $\lambda_{1}, \lambda_{2}, \lambda_{3}$. The normalized temperature $\tau=k_{B} T / U_{0}$ is along the $y$ axis and the angle $\vartheta$, measured from the point $A \equiv(0,0,1)$ in counter-clockwise direction, is along the $x$ axis. Compare to Fig.2.2.

of view put forward elsewhere that in some way the the passage across this phase diagram corresponds to an exchange of rods and discs, and avoids the puzzle of why there would be symmetry between the "rod" and "disc" section of the phase diagram.

Interestingly, however, a similar symmetric phase diagram has been obtained by Zhang and Pallfy-Muhoray [61], where the parameter analogous to our $\vartheta$ is connected to the molecular shape in a dispersive intermolecular interaction. In that case therefore, by contrast with the present study, the symmetry around $\vartheta=\pi / 3$ does indeed mark the passage from rod-like molecules to disc-like molecules.

2.4. Summary. The biaxial liquid crystal molecular field theory picture of Virga and coworkers (e.g. [56, 21, 12]) demonstrated that the uniaxial-biaxial liquid crystal phase diagram could be much richer than previously expected. In particular, it became clear that a regime of first-order isotropic-biaxial liquid crystal phase transitions was possible. A prominent part of this theory involved the reduction of the full set of biaxial parameters to a smaller essential set, which is pictured as an "essential triangle". Any other parameter was equivalent to one in the essential set by a group transformation.

We have reformulated this picture in a manner in which the interactions between different axes is given a more prominent role. The result is that the group theoretical structure involved in exchanging order parameters is much more transparent. The transformed essential triangle now transforms into an identical triangle, albeit rotated or reflected. As a parenthetical remark here, we note that the "dispersion parabola" of Ref.[21] is transformed to the geometric mean circle in our picture. Our triangles are related to the triangles of Ref.[21] through a projective transformation, which transforms one conic section (a parabola) to another (a circle) ${ }^{1}$.

The basic picture of Fig. 2.4 will be robust with respect to changes of intermolecular potential, so long as the basic $D_{2 h}$ symmetry of the molecules and the phases is retained. The picture will change, if for example, the system is constrained to a

${ }^{1}$ We are grateful to Professor E.G. Virga for this observation 
surface $[9,52]$, the principal axes are tilted with respect to each other, extra order parameters are required, and the low temperature symmetry of the phase is no longer $D_{2 h}$, as for example is the case in our recent study of $C_{2 h}$ systems [44].

The least robust feature of Fig. 2.4 is the small region marked in orange, in which there are successive first order transitions $I-N_{U}$ and $N_{U}-N_{B}$. It seems likely that changes in the parameterization of the interaction in mean field theory, or fluctuation effects in real systems, could cause the orange region to collapse entirely. Thus from the point of view of statistical mechanics, this narrow region, corresponding to a small parameter regime, presents an interesting challenge, both from a mathematical and an experimental point of view.

A second interesting regime corresponds to the outer region close to the edges of the "blue" triangle in Fig.2.3. In this region, the biaxial low temperature phase is only just stable. In real systems, this region will probably be pre-empted by other low temperature phases. But in model lattice systems, the multiphase lines are analogous to other multiphase lines in systems with competing interactions [34]. Thus the multiphase lines corresponding to this edge could exhibit rich structure, particularly if the nearest neighbor interactions are complemented by longer-range interactions with countervailing effects.

So far in this paper we have been concerned with global features of the phase diagram, rather than details of the dependence of order parameters on temperature. However, these details are also important in experiments. Furthermore, many features of the order parameter-temperature-biaxiality interdependence are amenable to analysis, and it is to this that we turn next.

3. Landau-like approximation for the ordered phases. In this section we shall be concerned primarily with the low temperature limit of molecular field theories of biaxial liquid crystals. This region is pre-empted by other more ordered phases in real liquids. It is however of considerable theoretical interest. In many cases, it is tempting to derive a phase diagram and temperature dependence of the order parameters computationally, without enquiring further into the details of the behavior. Analysis, however, gives insight into the results.

The derivation of the classical Landau-de Gennes free energy expansion is valid near the isotropic phase, close to the nematic-isotropic transition temperature. Therefore, it is well expected that the predictions are non-physical in the low-temperature regime; specifically, the order parameter $S$ is not constrained to reach one (perfect order), when the temperature goes to zero. Furthermore, for some values of the relative molecular biaxiality parameters, the order parameter $S$ is non-negligible close to the $N_{U}-N_{B}$ phase transition line. Therefore, $S$ possibly lies outside of the limits of validity of the Landau expansion and this fact questions the applicability of the classical Landau approximation to the study of the uniaxial-biaxial phase transition.

An alternative method to construct a (non-equilibrium) free energy density, is the KKLS method [36, 43]. It borrows the internal energy from the Maier-Saupe theory [48], but, unlike the molecular field theory, it derives the singlet orientational probability distribution from the maximum entropy principle [35, 33]. This result has been independently obtained by a number of authors [5, 6, 7]. A similar approach, in the context of Onsager-style models, was first derived by Colot et al [15] and rederived in somewhat simpler form by Fatkullin and Slastikov [24, 25].

This method has already been successfully applied to study the phase transitions in liquid crystals $[36,43,5,6]$. However, it has always been used as an alternative to the standard Landau approximation of the free energy. In such cases one performs 
a polynomial expansion of the probability distribution around an isotropic phase in which all the order parameters vanish.

In contrast, here we show that the validity of the KKLS approach is not limited to this case. Indeed, alternative approximations can be found which yield a Landau-like free energy that is valid in the high order regime $S \rightarrow 1$, i.e., at low temperatures. The final approximation to the free energy does not involve complicated integrals and its study only requires the minimization of a function with respect to the order parameters. What is lost, however, is the simple polynomial expression of the Landau free energy. As we shall see, a more complicated functional dependence must be considered, specifically a logarithmic dependence on the dominant order parameters (see also Ref.[7]).

\subsection{Introductory example: uniaxial molecules in a uniaxial phase.}

3.1.1. Free energy functional. Before embarking on the study of biaxial nematic liquid crystals, we present the method by calculating the Landau-like free energy, as derived in the KKLS protocol, for a system of uniaxial $\left(D_{\infty h}\right)$ molecules in a uniaxial $\left(D_{\infty h}\right)$ phase. This was the standard example used in the original Ref.[36], but, as opposed to what is done in [36], we now approximate the free energy in the high order phase.

The only necessary order parameter is $S$, as defined in Eq.(2.1). The average is performed with respect to the orientational probability function $f(\beta)$, where $\beta$ is the angle between the molecular symmetry axis and the director. The function $f(\beta)$ is derived from the maximum entropy principle, i.e., it is chosen as to maximize the entropy $\left(k_{B}\right.$ is the Boltzmann constant)

$$
\mathbb{S}=-k_{B} \int_{0}^{\pi} f(\beta) \log f(\beta) \sin \beta d \beta
$$

under the constraints

$$
S=\frac{1}{2}\left\langle 3 \cos ^{2} \beta-1\right\rangle, \quad \int_{0}^{\pi} f(\beta) \sin \beta d \beta=1 .
$$

Here the angle brackets mean the ensemble average with respect to $f$ :

$$
\langle\psi\rangle=\int_{0}^{\pi} \psi(\beta) f(\beta) \sin \beta d \beta .
$$

The general form of the orientational distribution function is that of the MaxwellBoltzmann probability distribution [35, 33]. In our specific example this is

$$
f(\beta, \eta)=\frac{1}{Z(\eta)} \mathrm{e}^{\eta\left(3 \cos ^{2} \beta-1\right) / 2}
$$

where $Z(\eta)$ is the partition function

$$
Z(\eta)=\int_{0}^{\pi} \mathrm{e}^{\eta\left(3 \cos ^{2} \beta-1\right) / 2} \sin \beta d \beta,
$$

and $\eta$ is the Lagrangian multiplier associated with the constraint in Eq.(3.2) for fixed $S$. The entropy is then calculated in terms of both $S$ and $\eta$ as

$$
\mathbb{S}=-k_{B}[\eta S-\log Z(\eta)] .
$$


The Lagrangian multiplier $\eta$ is related to the order parameter via the identity

$$
S=\frac{\partial \ln Z(\eta)}{\partial \eta},
$$

which, in principle, can be inverted to give $\eta$ as a function of $S$. The function $\eta=\eta(S)$ can then be inserted in (3.6) to give an expression of the entropy uniquely in terms of the order parameter $S$. In practice, without the introduction of special functions, the inversion of Eq.(3.7) can be performed only approximately and even then only in limited regions of the whole range $\eta \in[0,+\infty)$.

\subsubsection{Mathematical remarks.}

(i) The entropy, as given in (3.6), is proportional to the Legendre transform [37] of the function $\log Z(\eta)$, where the conjugate variables are $\eta$ and $S$. The involutive property of the Legendre transform then yields

$$
\frac{\partial \mathbb{S}}{\partial S}=-k_{B} \eta
$$

The Lagrangian multiplier $\eta$ is thus a "reactive force" originated by the constraint Eq.(3.2) for fixed $S$ [37].

(ii) Once the dependence of the Lagrangian multiplier on $S$ is known, Eq.(3.8) can be integrated to give the entropy as a function of $S$. This is of course an alternative procedure with respect to Eq.(3.6) to obtain the entropy. This is the key step enabling the free energy to be expressed as a function of $S$, and not merely a functional of $f(\beta)$.

(iii) In more complicated cases, when multiple order parameters (and therefore also multiple Lagrangian multipliers) are introduced, say $S_{i}, \eta_{i}, i=1 \ldots n$, Eq.(3.8) implies that the following compatibility conditions must hold:

$$
\frac{\partial \eta_{i}}{\partial S_{j}}=\frac{\partial \eta_{j}}{\partial S_{i}}
$$

(iv) In the standard applications of the method, the partition function is expanded around $\eta=0$ and then a series expansion of $S$ in terms of powers of $\eta$ is obtained from Eq.(3.7). This expansion is then inverted [1] to give the Taylor expansion of $\eta$ in terms of powers of $S$. The leading order of this expansion is $\eta \sim S$, thus indicating that expanding $Z(\eta)$ around $\eta=0$ corresponds to the high temperature approximation of the free energy $(S \approx 0)$. This is consistent with the idea of a Lagrangian multiplier being a "reactive force" that imposes the constraint. When $S \approx 0$, the probability distribution is close to being uniform and thus the entropy is close to its free maximum, where no constraint is actually needed. This approximation is expected to be accurate, and indeed it is [43], only close to the $I-N_{U}$ phase transition.

(v) By contrast, in the limit $S \rightarrow 1$ (high order phase), the constraint must impose an infinite "reactive force" in order to obtain a probability distribution far from the entropy-free maximum. Imposing $S \rightarrow 1$ selects $z$ as the preferred molecular and laboratory axes, which may seem contrary to the spirit of the symmetry-preserving representation of the phase sequences given by the ternary diagram discussed in Sec.2. However, for some practical purposes it is indeed helpful to use coordinates. The use of the ternary diagram easily allow us to divide the biaxiality parameter plane into equivalent regions and 
therefore the results obtained for a particular choice of preferred axes can be easily mapped into equivalent results in the other regions. Once this symmetry is clearly understood and easily accessible there is no intrinsic restriction in assigning a preferred status to any of the molecular and laboratory axes.

3.1.3. Low temperature expansion of the Maier-Saupe theory. As a new application of the method, we seek an asymptotic approximation of the partition function, as $\eta \rightarrow+\infty$. To this end, we apply the Laplace method [8] to the integral (3.5) as $\eta \rightarrow+\infty$. To leading order we find

$$
Z(\eta) \sim \frac{2 \mathrm{e}^{\eta}}{3 \eta}
$$

Using the identity (3.7) we derive $S=(\eta-1) / \eta$, which is then easily inverted to give $\eta=1 /(1-S)$. Neglecting the irrelevant constants in the entropy, Eq.(3.6) or Eq.(3.8) then yield

$$
\mathbb{S}=k_{B} \log (1-S)
$$

With the purpose of writing the free energy, we use the internal energy as derived from the molecular field theory and simplify Eq.(2.3) to give

$$
U=-\frac{u_{200}}{2} S^{2}
$$

Eq.(3.12) is consistent at equilibrium with the classic Maier-Saupe expression, which is used to construct the equilibrium free energy in a molecular field approximation. Finally, we arrive at the following expression for the free energy difference per particle,

$$
\mathscr{F}=U-T \mathbb{S}=-\frac{u_{200}}{2} S^{2}-k_{B} T \log (1-S),
$$

where $T$ is the absolute temperature.

The presence of a logarithmic singularity at $S=1$ constrains the order parameter $S$ to remain below its limiting value of unity at perfect order. As such it is expected; this idea has been explored from a slightly different point of view by Ball and Majumdar [7]. This is to be contrasted with standard Landau expansions of the free energy $(S \approx 0)$, for which there is no upper bound for the predicted order parameter $S$ at low temperature. In the low $S$ limit this is, of course, an unimportant constraint. The equilibrium order parameter obtained from the minimization of Eq.(3.13) is calculated to be $S=\left(1+\sqrt{1-4 k_{B} T / u_{200}}\right) / 2$. We then verify directly that $S \leq 1$, for all temperatures.

\subsection{Biaxial molecules in a biaxial phase.}

3.2.1. Theory. We now apply the KKLS procedure to the study of a system composed of biaxial $\left(D_{2 h}\right)$ molecules in a biaxial nematic $\left(D_{2 h}\right)$ phase. The approximation now entails all the four order parameters $S, P, D$ and $C$ as defined in Eqs. (2.1), (2.2), where the average is performed over all possible Euler angles $\alpha, \beta$ and $\gamma$ :

$$
\langle\psi\rangle=\int_{0}^{\pi} \sin \beta d \beta \int_{0}^{2 \pi} d \alpha \int_{0}^{2 \pi} d \gamma \psi(\alpha, \beta, \gamma) .
$$

The partition function is

$$
Z(\eta)=\left\langle\exp \left[\eta_{S} \psi_{S}(\beta)+\eta_{P} \psi_{P}(\beta, \gamma)+\eta_{D} \psi_{D}(\alpha, \beta)+\eta_{C} \psi_{C}(\alpha, \beta, \gamma)\right]\right\rangle .
$$


The functions $\psi_{S}, \psi_{P}, \psi_{D}$ and $\psi_{C}$ are defined as the angular functions in Eqs.(2.1),(2.2) whose averages yield the order parameters $S, P, D$ and $C$, respectively. Our purpose is to write a Landau-like free energy that correctly describes the uniaxial-to-biaxial phase transition, when it occurs at low temperatures. Specifically we suppose the transition to appear in the nearly perfectly aligned uniaxial phase. Implicitly we also suppose weak molecular biaxiality.

We thus now seek an approximate expression of the partition function assuming that the system is initially in a well-ordered uniaxial nematic phase, with $S \approx 1$ and $C \approx 0$. Moreover, the condition $S \approx 1$ implies small values for the minor order parameters, therefore we can also assume $P \approx 0$ and $D \approx 0$.

REMARK 3.1. This is not the only possible choice. We could choose $S \approx 1$ and $C \approx 3$. This yields the approximation to the free energy in a nearly perfectly ordered biaxial nematic phase. Minimization of this free energy then yields the low temperature behavior of the minor order parameters $P, D$.

This approximation corresponds to evaluating the integrals in Eq.(3.15) in the limit $\eta_{S} \rightarrow+\infty$ and $\eta_{C} \approx 0$. We therefore apply the Laplace method with respect to $\eta_{S}$. To be able to include the effects of the minor order parameters, we do not limit the expansion to the leading order, but we carry it up to $O\left(\beta^{6}\right)$, yielding

$$
\begin{aligned}
Z & =\mathrm{e}^{\eta_{S}} I_{0}\left(3 \eta_{C}\right)\left[\frac{1}{3 \eta_{S}}+\frac{1}{9 \eta_{S}^{2}}+\frac{1}{405 \eta_{S}^{3}}+\frac{\eta_{P}^{2}+\eta_{D}^{2}+\eta_{C}^{2}}{6 \eta_{S}^{3}}\right] \\
& +\mathrm{e}^{\eta_{S}} I_{1}\left(3 \eta_{C}\right)\left[-\frac{\eta_{C}}{3 \eta_{S}^{2}}+\frac{\eta_{C}}{6 \eta_{S}^{3}}+\frac{\eta_{D} \eta_{P}}{3 \eta_{S}^{3}}\right]+\mathrm{e}^{\eta_{S}} I_{2}\left(3 \eta_{C}\right) \frac{\eta_{C}^{2}}{6 \eta_{S}^{3}}+o\left(\frac{\mathrm{e}^{\eta_{S}}}{\eta_{S}^{3}}\right)
\end{aligned}
$$

where $I_{\nu}(z)$ is the modified Bessel function of the first kind [1].

The relations between the order parameters and the Lagrangian multipliers, analogous to Eq.(3.7), yield the series expansion of the order parameters in terms of $1 / \eta_{S}$, $\eta_{P}, \eta_{D}$ and $\eta_{C}$. The inversion of these expansion, to give the dependence of the Lagrangian multipliers in terms of the order parameters, requires some extra care with respect to the uniaxial case studied in Sec.3.1. However, with the aid of Eq.(3.9), the leading asymptotic behavior is found to be

$$
\begin{aligned}
\eta_{S} & \sim \frac{1-S}{(1-S)^{2}-\left(P^{2}+D^{2}\right)}, & \eta_{P} & \sim \frac{P}{(1-S)^{2}-\left(P^{2}+D^{2}\right)}, \\
\eta_{D} & \sim \frac{D}{(1-S)^{2}-\left(P^{2}+D^{2}\right)}, & \eta_{C} & \sim \frac{2}{9} C .
\end{aligned}
$$

After some algebra, which we omit for brevity, the higher terms of the inversion can also be identified. By integration is easy to show the leading contribution to the entropy is, as for the uniaxial case of Sec.3.1, a logarithmic function:

$$
\mathbb{S} \sim \frac{k_{B}}{2} \log \left[(1-S)^{2}-\left(P^{2}+D^{2}\right)\right]
$$

Finally, following the KKLS protocol outlined in Sec.3.1 and using the internal energy as given in Eq.(2.13) leads to the free energy expansion,

$$
\begin{aligned}
\mathscr{F} / U_{0} & =-\frac{1}{3}\left[\left(\frac{\lambda_{1}+\lambda_{2}}{4}+\lambda_{3}\right)\left(S^{2}+\frac{1}{3} P^{2}\right)+\frac{1}{2}\left(\lambda_{2}-\lambda_{1}\right)\left(S D+\frac{1}{3} P C\right)\right. \\
& \left.+\frac{1}{4}\left(\lambda_{1}+\lambda_{2}\right)\left(D^{2}+\frac{1}{3} C^{2}\right)\right]-\tau\left\{\frac{1}{2} \log \left[(1-S)^{2}-\left(P^{2}+D^{2}\right)\right]\right. \\
& \left.-\frac{C^{2}}{9}-\frac{C^{4}}{324}-\frac{2}{27} C^{2} \frac{(1-S)^{2}-\left(P^{2}+D^{2}\right)}{1-S}\right\},
\end{aligned}
$$


where $\tau=k_{B} T / U_{0}$ is the reduced temperature.

In writing the free energy (3.20) we have omitted for simplicity the polynomial part of $(1-S)$ since it gives only small corrections to the entropy. Indeed, the logarithmic part dominates the entropy in the limit of uniaxial order $S \approx 1, P \approx 0$, $D \approx 0$. By contrast and as a consequence of our approximation, the free energy has only a polynomial dependence on the order parameter $C$ and at least the fourth degree must be retained in order to study the $N_{U}-N_{B}$ transition. The last term of Eq.(3.20) is the lowest degree term that couples the biaxial order parameters $C$ with $S$. Of course, more elaborate expansions for the free energy could be obtained by keeping higher order terms. However, this could be done only at the cost of greatly complicate the free energy and with a little gain for the physical insight.

\subsubsection{Applications.}

(i) Order parameter properties in a uniaxial $\left(N_{U}\right)$ phase of biaxial $\left(D_{2 h}\right)$ molecules. The relevant order parameters in this case are $S$ and $D$, since by assumption $P=C=0$. The equilibrium order parameters are critical points of the free energy (3.20) and hence solve the two equations $\frac{\partial \mathscr{F}}{\partial S}=0$ and $\frac{\partial \mathscr{F}}{\partial D}=0$. The reduced temperature, $\tau$, can be eliminated from these equations to yield the following equation for the order parameters:

$$
\lambda_{1}(D-S)(1-S+D)+\lambda_{2}(1-S-D)(D+S)-4 \lambda_{3} S D=0 .
$$

This equation can be used to calculate $D$ as a function of $S$ at equilibrium. The leading approximation to the solution of Eq.(3.21), as $S \rightarrow 1$, is

$$
D=\frac{\lambda_{2}-\lambda_{1}}{\lambda_{1}+\lambda_{2}+4 \lambda_{3}}(1-S)+o\left((1-S)^{2}\right) .
$$

A few comments on these results are now in order.

REMARK 3.2 .

(a) We have shown rigorously that $D \rightarrow 0$ as $S \rightarrow 1$. This is physically intuitive from the definition of $S$ and $D$. In Eq.(2.1), $S \approx 1$ implies $\beta \approx 0$ and hence in $E q .(2.2) D \approx 0$.

(b) We further find that $D \sim(1-S)$ at very low temperature.

(c) Along the Sonnet-Virga-Durand line, where $\lambda_{1}=\lambda_{2}$ and the $x$ and $y$ molecular axes are equivalent, $D$ vanishes identically.

(ii) Can a system of uniaxial $D_{\infty h}$ molecules posses a $N_{B}$ phase? Intuitively this seems unlikely, but it is desirable to see this explicitly in the mathematical description. To this end, we set $D=C=0$ in Eq.(3.20). The minimization of the free energy then immediately yields $P=0$. Therefore, the KKLSderived free energy prohibits a $N_{B}$ phase for uniaxial molecules, even at low temperature. This is to be contrasted with at least formal predictions of standard Landau theories [17, 29] and molecular field theories [45].

(iii) The $N_{U}-N_{B}$ phase boundary line along the Sonnet-Virga-Durand line. Within this approximation, the critical points of $\mathscr{F}$ have $P=D=0$. The only "essential" variables in this case are $S$ and $C$. We set $\lambda=\lambda_{1}=\lambda_{2}$ and $\lambda_{3}=1-2 \lambda$. The phase line is determined by the loss of stability of the uniaxial minimum. We therefore write the two following equations in the three unknowns $S, \lambda$ and $\tau$, and evaluated at $P=D=C=0$ :

$$
\frac{\partial \mathscr{F}}{\partial S}=0, \quad \frac{\partial^{2} \mathscr{F}}{\partial C^{2}}=0 .
$$


The first equation is the equilibrium condition for the uniaxial order parameter $S$. The second equation expresses the bifurcation condition valid for a second-order transition to the $N_{B}$ phase. The order parameter $S$ can now be easily eliminated from Eqs.(3.23) to yield an implicit expression of the transition line, in terms of $\lambda$ and $\tau$ :

$$
16 \tau^{3}+(2-3 \lambda)(3 \lambda-10 \tau)(\lambda-2 \tau)=0
$$

It is known from [56] that along this line there is a tricritical point, where the transition $N_{U}=N_{B}$ becomes first order. To locate the tricritical point we resort to the method described in Ref.[21, 28]. This entails the solution of the additional equation

$$
\frac{\partial^{2} \mathscr{F}}{\partial S^{2}} \frac{\partial^{4} \mathscr{F}}{\partial C^{4}}-3\left(\frac{\partial^{3} \mathscr{F}}{\partial S \partial C^{2}}\right)^{2}=0,
$$

evaluated at $P=D=C=0$. Eqs.(3.23) and (3.25) are three equations in the unknowns $S, \lambda$ and $\tau$ which can be solved numerically to give the location of the tricritical point, yielding $\left(\lambda_{T}, \tau_{T}\right) \approx(0.26,0.10)$. This region is close to the $N_{U}-I$ transition, where our approximation would not normally be expected to yield accurate results. But remarkably, the estimated position of the tricritical point is in rather good agreement with the numerical results of the mean-field theory described in Ref.[56].

4. Discussion. We first look at the context of our study. The study of phase transitions in thermotropic nematic biaxial liquid crystals has attracted much theoretical and experimental work over recent decades. Nevertheless a complete understanding of this phenomenon remains elusive, as the continuing debate over recent relevant experimental results $[47,2]$ testifies. Following as a consequence of this debate, the experimental rarity of the $N_{B}$ phase has come as a surprise to the theoretical community. It seems likely that the theoretical models remain inadequate. However, it is possible that insufficient theoretical attention has been paid to competition with crystalline phases. It may also be that some future insight will make the synthesis of biaxial phases much easier.

We now turn the content of the paper itself. Theoretical studies of biaxial phases, even in the context of the molecular field theory, have employed a wide range of notations. These seem to have been mainly motivated by the personal taste of the authors [54], rather than by any overarching strategy. The first part of this paper demonstrates, however, that the symmetry of the system dictates a natural choice of biaxiality parameters. The key advantages of the representation which we have introduced are a clearer visualization of the various phase diagram and the easy exploitation of the symmetry. It is in fact straightforward to identify the regions of the biaxiality parameter space where the system has equivalent physical behavior. In this way all the known results in the literature are unified under a common framework and their intimate connections are revealed. In other words, we have shown that, in order to obtain a clean, symmetry preserving, description of the phase diagram, the group theoretical structure involved in exchanging order parameters must be explicitly considered when choosing molecular biaxial parameters.

In the second part of the paper we have derived a Landau-like expansion of the free energy that can accurately describe the system in an ordered phase, where, by contrast, the validity of standard Landau theory is questionable. In doing so, we 
have followed the maximum entropy principle, first outlined in the context of liquid crystals almost 25 years ago [36], and rediscovered independently in Refs. [7, 5, 6]. The advantages of our method over the standard Landau theory are thoroughly discussed in [43]. The resulting low-temperature approximation is non-polynomial in the order parameters. This, however, is to be contrasted with the standard Landau theories, where the free energy is constructed form the polynomial invariants.

We have shown that the non-polynomial nature of the free energy is essential in order to predict the correct limiting values of the order parameters in the ordered phases. These limiting values are neglected in the standard Landau approximation because this is the truncation of a polynomial expansion, e.g., the order parameter $S$ is not constrained to go to 1 , as $\tau \rightarrow 0$ [50]. This implies that the strategy adopted in the Landau expansions, i.e., build the free energy taking a linear combination of all the polynomial invariants with phenomenological coefficients, must fail because the low temperature expansion of the free energy necessarily comprises non-polynomial invariants (now of a subgroup of the original symmetry group), specifically in our case, a logarithmic invariant.

We have therefore presented a procedure, where the internal energy is taken from the molecular field theory and the entropy is derived from the maximum entropy principle. This yields a Landau-like approximation of the free energy which is consistent with the physical bounds. The procedure is simple enough to perform an analysis of the order parameters analytically or semi-analytically. As examples of applications we have derived the dependence of the minor order parameters, $P$ and $D$, on the major order parameter $S$ and on molecular shape. We have also been able, using low temperature extrapolations alone, to estimate the existence and position of the $N_{U}-N_{B}$ tricritical point, which was a surprise when first derived theoretically.

Acknowledgements. The authors would particularly like to thank Professor Geoffrey Luckhurst for his encouragement and for many discussions; he derived the key result Eq.(2.15). They also thank P. Biscari, F. Bisi, D.R.J. Chillingworth, E.C. Gartland, L. Longa, R. Rosso and E.G. Virga for useful conversations and correspondence. They also acknowledge financial support from the Royal Society of London through International Joint Project grant IJP-2004/R1-EU: Biaxial nematic liquid crystals: Mathematical models and simulation, 20042007. S.S.T. is grateful to the Schools of Chemistry and Mathematics at the University of Southampton for fellowship support and hospitality during periods over which some of this work was carried out.

\section{REFERENCES}

[1] M. Abramowitz and I. Stegun, Handbook of Mathematical Functions with Formulas, Graphs, and Mathematical Tables, Dover, New York, 1965.

[2] B. R. Acharya, A. Primak, and S. Kumar, Biaxial nematic phase in bent-core thermotropic mesogens, Phys. Rev. Lett., 92 (2004), p. 145506.

[3] R. Alben, Phase transitions in a fluid of biaxial particles, Phys. Rev. Lett., 30 (1973), p. 778.

[4] L. Q. Amaral, First-order transition between nematic phases in lyotropic liquid crystals, Liq. Cryst., 7 (1990), p. 877.

[5] E. M. Aver'yanov, Structural and optical anisotropy of biaxial nematics, J. Struct. Chem+, 42 (2001), p. 598.

[6] - Fluctuations of orientational order in a uniaxial nematic liquid crystal with biaxial molecules and its response to an external field, Phys. Solid State+, 45 (2003), p. 990.

[7] J. M. Ball And A. Majumdar, Nematic liquid crystals: From Maier-Saupe to a continuum theory, Mol. Cryst. Liq. Cryst., 525 (2010), pp. 1-11. 
[8] C. M. Bender and S. A. Orszag, Advanced Mathematical Methods for Scientists and Engineers: Asymptotic Methods and Perturbation Theory, McGraw-Hill, New York, 1978.

[9] P. Biscari, G. Napoli, and S. Turzi, Bulk and surface biaxiality in nematic liquid crystals, Phys. Rev. E, 74 (2006), p. 031708.

[10] F. Biscarini, C. Chiccoli, P. Pasini, F. Semeria, and C. Zannoni, Phase diagram and orientational order in a biaxial lattice model: A Monte Carlo study, Phys. Rev. Lett., 75 (1995), p. 1803.

[11] F. Bisi, G. R. LuCKhurst, And E. G. Virga, Dominant biaxial quadrupolar contribution to the nematic potential of mean torque, Phys. Rev. E, 78 (2008), p. 021710.

[12] F. Bisi, E. G. Virga, E. C. Gartland, Jr., G. De Matteis, A. M. Sonnet, and G. E. DuRAND, Universal mean-field phase diagram for biaxial nematics obtained from a minimax principle, Phys. Rev. E, 73 (2006), p. 051709.

[13] N. Boccara, R. Mejdani, and L. D. Seze, Solvable model exhibiting a first-order phase transition, J. de Phys., 38 (1977), p. 149.

[14] D. R. J. Chillingworth, Critical points and symmetries of a free energy function for biaxial liquid crystals. To be published.

[15] J.-L. Colot, X.-G. Wu, H. Xu, and M. Baus, Density-functional, landau and onsager theories of the isotropic-nematic transition, Phys. Rev. A, 38 (1988), p. 2022.

[16] H. S. M. Coxeter, Introduction to Geometry, John Wiley and Sons, New York, 2nd. ed., 1969.

[17] P. De Gennes and J. Prost, The Physics of Liquid Crystals, Oxford University Press, Oxford, 2nd. ed., 1995.

[18] G. De Matteis, F. Bisi, And E. G. Virga, Constrained stability for biaxial nematic phases, Continuum Mech. Thermodyn., 19 (2007), pp. 1-23.

[19] G. De Matteis and S. Romano, Mesogenic lattice models with partly antinematic interactions producing uniaxial nematic phases, Phys. Rev. E, 80 (2009), p. 031702.

[20] G. De Matteis, S. Romano, and E. G. Virga, Bifurcation analysis and computer simulation of biaxial liquid crystals, Phys. Rev. E, 72 (2005), p. 041706.

[21] G. De Matteis and E. G. Virga, Tricritical points in biaxial liquid crystal phases, Phys. Rev. E, $71(2005)$, p. 061703.

[22] D. A. Dunmur and K. Toriyama, Tensor properties of anisotropic materials, Wiley-VCH, Weinheim, 1999, ch. IV, p. 87.

[23] U. Fabbri And C. Zannoni, A Monte Carlo investigation of the Lebwohl-Lasher lattice model in the vicinity of its orientational phase transition, Mol. Phys., 58 (1986), pp. 763-788.

[24] I. Fatkullin and V. Slastikov, On spatial variations of nematic ordering, Phys. D, 237 (2008), pp. 2577-2586.

[25] — Vortices in two-dimensional nematics, Commun. Math. Sci., 7 (2009), pp. 917-938.

[26] M. J. Freiser, Ordered states of a nematic liquid, Phys. Rev. Lett., 24 (1970), pp. 1041-1043.

[27] — Successive transitions in a nematic liquid, Mol. Cryst. Liq. Cryst., 14 (1971), p. 165.

[28] L. Gibelli AND S. TURZI, A catastrophe-theoretic approach to tricritical points with application to liquid crystals, SIAM J. Appl. Math., 70 (2009), p. 63.

[29] F. H. Gramsbergen, L. Longa, , and W. H. DE JeU, Landau theory of the nematic-isotropic phase transition, Phys. Rep., 135 (1986), p. 195.

[30] F. GRandjean, Sur l'application de la théorie du magnétisme aux liquides anisotropes, Comptes Rendus de l'Académie des Sciences, 164 (1917), p. 280.

[31] C. G. Gray and K. E. Gubbins, Theory of Molecular Fluids, Oxford University Press, Oxford, 1984.

[32] G. S. Grest and E. G. Gabl, Monte Carlo study of spin-glass ordering on dilute frustrated lattices, Phys. Rev. Lett., 43 (1979), p. 1182.

[33] H. HAKen, Information and Self-Organization: A Macroscopic Approach to Complex Systems, Springer-Verlag, Berlin, 1988.

[34] D. A. Huse, M. E. Fisher, And J. M. Yeomans, Multiphase behavior and modulated ordering in soluble Ising models, Phys. Rev. B, 23 (1981), pp. 180-185.

[35] E. T. JAYNeS, Information theory and statistical mechanics, Phys. Rev., 106 (1957), p. 620.

[36] J. Katriel, G. F. Kventsel, G. R. Luckhurst, and T. J. Sluckin, Free energies in the Landau and molecular field approaches, Liq. Cryst., 1 (1986), p. 337.

[37] C. Lanczos, The Variational Principles of Mechanics, Dover, New York, 1986. Reprint of 1970 original.

[38] P. A. Lebwohl and G. Lasher, Nematic-liquid-crystal order-a Monte Carlo calculation, Phys. Rev. A, 6 (1972), p. 426.

[39] G. R. Luckhurst, Molecular field theories of nematics, Academic Press, 1979, ch. 4.

[40] — Biaxial nematic liquid crystals: fact or fiction?, Thin Solid Films, 40 (2001), p. 393.

[41] - Liquid crystals: a missing phase found at last?, Nature, 430 (2004), p. 413. 
[42] — Biaxial nematics composed of flexible molecules: a molecular field theory, Liq. Cryst., 36 (2009), p. 1295.

[43] G. R. Luckhurst, S. Naemura, T. J. Sluckin, K. Thomas, and S. Turzi, Molecular-fieldtheory approach to the Landau theory of liquid crystals: Uniaxial and biaxial nematics, Phys. Rev. E, 85 (2012), p. 031705.

[44] G. R. Luckhurst, S. Naemura, T. J. Sluckin, T. B. T. To, And S. S. Turzi, Molecular field theory for biaxial nematic liquid crystals composed of molecules with $C_{2 h}$ point group symmetry, Phys. Rev. E, 84 (2011), p. 011704.

[45] G. R. Luckhurst and T. B. T. To. Private communication, 2012.

[46] G. R. Luckhurst, C. Zannoni, P. L. Nordio, and U. Segre, A molecular field theory for uniaxial nematic liquid crystals formed by non-cylindrically symmetric molecules, Mol. Phys., 30 (1975), p. 1345.

[47] L. A. Madsen, T. J. Dingemans, M. Nakata, and E. T. Samulski, Thermotropic biaxial nematic liquid crystals, Phys. Rev. Lett., 92 (2004), p. 145505.

[48] W. Maier and A. Saupe, A simple molecular theory of the nematic crystalline-liquid state, Z. Naturforsch., 13a (1958), p. 564. (see Crystals that Flow, ed. T.J. Sluckin, D.A. Dunmur and H. Stegemeyer (Taylor and Francis, London 2004) for English translations of Refs $[59,30,48]$.$) .$

[49] G. C. Maitland, M. Rigby, E. B. Smith, and W. A. Wakeham, Intermolecular Forces, Clarendon, Oxford, 1981.

[50] A. Majumdar, Equilibrium order parameters of nematic liquid crystals in the Landau-de Gennes theory, Eur. J. Appl. Math., 21 (2010), p. 181.

[51] B. Mulder, Isotropic-symmetry-breaking bifurcations in a class of liquid-crystal models, Phys. Rev. A, 39 (1989), p. 360.

[52] G. Napoli and L. Vergori, Surface free energies for nematic shells, Phys. Rev. E, 85 (2012), p. 061701

[53] P.-O. Quist, First order transitions to a lyotropic biaxial nematic, Liq. Cryst., 18 (1995), p. 623.

[54] R. Rosso, Orientational order parameters in biaxial nematics: Polymorphic notation, Liq. Cryst., 34 (2007), p. 737.

[55] J. S. Rowlinson And F. L. Swinton, Liquids and Liquid Mixtures, Butterworths, London, 3rd. ed., 1982.

[56] A. M. Sonnet, E. G. Virga, and G. E. Durand, Dielectric shape dispersion and biaxial transitions in nematic liquid crystals, Phys. Rev. E, 67 (2003), p. 061701.

[57] J. P. Straley, Ordered phases of a liquid of biaxial particles, Phys. Rev. A, 10 (1974), p. 1881.

[58] S. S. TuRzI, On the cartesian definition of orientational order parameters, J. Math. Phys., 52 (2011), p. 053517.

[59] D. Vorländer, Über krystallinisch-flüssige substanzen, Berichte der Deutschen Chemischen Gesellschaft, 39 (1906), p. 803.

[60] L. J. YU AND A. SAUPE, Observation of a biaxial nematic phase in potassium laurate-1-decanolwater mixtures, Phys. Rev. Lett., 45 (1980), p. 1000.

[61] X. Zheng And P. PalfFy-Muhoray, One order parameter tensor mean field theory for biaxial liquid crystals, Discret. Contin. Dyn. S., 15 (2011), p. 475. 